\title{
Relação entre Sintomatologia Psicopatológica, Adesão ao Tratamento e Qualidade de Vida na Infecção HIV e AIDS
}

\author{
Relationship among Psychopathological Symptoms, Treatment Adherence \\ and Quality of Life in HIV/AIDS Infection
}

\author{
Ana Catarina Reis ${ }^{a}$, Leonor Lencastre ${ }^{a}$, Marina Prista Guerra ${ }^{*}, a$ \& Eduardo Remor ${ }^{b}$ \\ ${ }^{a}$ Universidade do Porto, Porto, Portugal \& ${ }^{b}$ Universidad Autónoma de Madrid, Madri, Espanha
}

\begin{abstract}
Resumo
O presente estudo observou a existência de relações significativas entre sintomatologia psicopatológica (BSI), adesão ao tratamento (CEAT-VIH) e qualidade de vida (WHOQOL-Bref) em 125 pessoas HIVpositivo/AIDS em tratamento antirretroviral, atendidos em um hospital do Porto (Portugal). A sintomatologia psicopatológica correlacionou-se negativamente com adesão ao tratamento e qualidade de vida, e a adesão ao tratamento associou-se positivamente com a qualidade de vida. O modelo preditor de adesão ao tratamento, mediante análise de regressão múltipla, incluiu a ausência de sintomatologia psicopatológica, o comportamento de retirada dos medicamentos na farmácia hospitalar e a presença de efeitos colaterais do tratamento $\left(R^{2}=0,30\right)$. Ademais, observou-se um efeito mediador de sintomatologia psicopatológica entre a adesão ao tratamento e a qualidade de vida das pessoas com HIV/AIDS.
\end{abstract}

Palavras-chave: Adesão ao Tratamento; HIV/AIDS; CEAT-VIH; Qualidade de Vida; Saúde Mental.

\begin{abstract}
The study shows evidence of statistical association among psychopathological symptoms (BSI), treatment adherence (CEAT-VIH) and quality of life (WHOQOL-Bref) in $125 \mathrm{HIV}$-positive/AIDS patients followed at Porto Hospital (Portugal). The psychopathological symptoms present a negative correlation with treatment adherence and quality of life. On the other hand, adherence was positively correlated with quality of life. The model of adherence to the antiretroviral treatment using multiple regression analysis includes absence of psychopathological symptoms, an adherence behavior (i.e. to get the medication at the hospital's pharmacy) and the experience of side effects because of medication $\left(R^{2}=0.30\right)$. Besides, psychopathological symptoms have a mediation effect between adherence and quality of life in people with HIV/AIDS.

Keywords: Treatment Adherence; HIV/AIDS; CEAT-VIH; Quality of Life; Mental Health.
\end{abstract}

Após a década de 1970, quando se julgavam controladas as maiores doenças infecciosas, o mundo foi assolado por um novo problema de saúde, que mais tarde foi denominado de Infecção pelo Vírus da Imunodeficiência Humana (HIV). A infecção pelo HIV é uma doença complexa de curso lento e incerto que leva o indivíduo a desenvolver a Síndrome da Imunodeficiência Adquirida (AIDS). A infecção pelo HIV, pelas suas características particulares de transmissão, atinge proporções preocupantes até os dias de hoje, e é considerada pela Organização Mundial de Saúde como uma pandemia. Em 1996 surgiu uma nova opção de tratamento farmacológico antirretroviral (Inibidores da Protease) que trouxe espe-

* Endereço para correspondência: Universidade do Porto, Faculdade de Psicologia e de Ciências da Educação, Rua Dr. Manuel Pereira da Silva, Porto, Portugal, 4200-392. Email:mguerra@fpce.up.pt rança aos portadores do vírus. A combinação dos inibidores da protease com os anteriores antiretrovirais possibilitou o desenvolvimento de um tratamento antirretroviral de alta atividade mais eficaz, que promoveu a infecção pelo HIV e AIDS ao estatuto de doença crônica, controlável a longo prazo, ou seja, permitindo a diminuição de episódios mórbidos e frequência de internamentos, e com consequente aumento da sobrevivência e melhoria da qualidade de vida (Canavarro, Pereira, Simões, Pintassilgo, \& Ferreira, 2008; Remor, 2003a; Siegel \& Lekas, 2002).

Porém, para alcançar estes benefícios e manter a infecção controlada é fundamental um grau de adesão à terapêutica superior a 90\% (Remor, 2002a; Remor, Milner-Moskovics, \& Preussler, 2007). Devido à complexidade do tratamento, muitos pacientes referem dificuldades para cumprir as prescrições, requerendo intervenção e apoio psicológico para superá-las ou melhorar 
o grau de adesão ao tratamento (Remor, 2003b; Remor, Ballester-Arnal, Espada-Sánchez, \& Gordillo ÁlvarezValdés, 2008).

Com a descoberta de novos medicamentos, observouse um maior controle da progressão da infecção e, como consequência, a manutenção da saúde. Ademais da qualidade do tratamento farmacológico, outros indicadores, tais como o apoio social (Giovelli, 2009; Remor, 2002b) e o estresse (Remor, Penedo, Shen, \& Schneiderman, 2007), foram sistematicamente relacionados à qualidade de vida em pessoas portadoras de HIV. Para uma revisão consultar Remor et al. (2008).

De importância similar à qualidade de vida, a saúde mental do indivíduo portador de HIV pode ser prejudicada se este não utiliza estratégias adequadas de enfrentamento (coping) para lidar com a doença e com o estresse associado (Campos, Guimarães, \& Remien, 2008; Carrobles, Remor, \& Rodríguez-Alzamora, 2003). As alterações psicológicas mais comuns que surgem perante um diagnóstico positivo ao HIV são: estresse, ansiedade e depressão (Grilo, 2001; Guerra, 1998; Remor, 1999). Estas alterações podem constituir uma etapa passageira no processo de adaptação psicológica à doença e conhecimento do diagnóstico de HIV-positivo (Remor, 2003b) ou podem levar a quadros clínicos mais severos se o indivíduo não apresentar recursos pessoais ou psicológicos suficientes para superar as experiências adversas e negativas (Serovich, Kimberly, Mosack, \& Lewis, 2001).

Considerando a relevância da qualidade de vida e a implicação de fatores psicológicos diante de um diagnóstico positivo de HIV, este trabalho, que faz parte de um estudo mais amplo (Reis, 2007), tem como objetivo principal identificar se existe relação entre sintomatologia psicopatológica, adesão ao tratamento e qualidade de vida em pessoas portadoras de HIV e AIDS.

\section{Método}

\section{Participantes}

A amostra foi constituída por 125 participantes portugueses com diagnóstico de HIV-positivo, do sexo feminino $(n=24 ; 19,2 \%)$ e masculino $(n=101 ; 80,8 \%)$, e idades entre 20 e 68 anos $(M=39,9$ anos; $D P=9,8)$. Quanto ao estado civil, a amostra distribuiu-se em: 55 (44\%) solteiros/as, 50 (40\%) vivendo com parceiro/a, e $20(16 \%)$ separados/as, divorciados/as ou viuvos/as. No que se refere ao nível de escolaridade, os participantes apresentaram-se conforme a distribuição: um a quatro anos de estudo $(n=49 ; 39,2 \%)$, cinco a nove anos $(n=$ $56 ; 44 \%), 10$ a 12 anos ( $n=13 ; 10,4 \%)$, e nível universitário $(n=8 ; 6,4 \%)$. Referente à ocupação, $38,4 \%(n=$ 48 ) encontravam-se profissionalmente ativos, $32,8 \%$ ( $n$ $=41)$ desempregados, e 28,8\% ( $n=36)$ em outra situação (aposentadoria ou baixa médica).

De acordo com os dados apresentados na Tabela 1, a via de infecção predominante foi através de relações sexuais $(62,4 \%)$. $\mathrm{O}$ esquema antirretroviral de tratamento prescrito na maioria dos casos $(39,2 \%)$ foi a combinação dos Inibidores Nucleósidos da Transcriptase Reversa com Inibidores Não Nucleósidos da Transcriptase Reversa. O tempo médio de infecção pelo HIV foi de 69,5 meses $(D P=41,6)$ e variou no intervalo de quatro a 170 meses.

No que se refere aos indicadores clínicos da infecção HIV/AIDS, os valores médios da carga viral situaram-se em 2029,8 cópias/ml de vírus no sangue $(D P=7575,9)$, sendo o mínimo $<50$ (abaixo do limiar de detecção) e o máximo 57.698. Do total dos participantes que integraram a amostra, 70,4\% apresentaram valores de carga viral indetectáveis. Para a contagem de linfócitos T CD4+, o valor médio encontrado foi de 359,7 CD4+ $(D P=$ $243,1)$, variando entre 14 e 1167 . Do total dos sujeitos que compuseram a amostra, $24,8 \%$ apresentaram contagem de linfócitos T CD4+ menor que 200 (critério para diagnóstico de AIDS).

Ainda, foi avaliado o grau de adesão dos participantes a retirada da medicação antirretroviral na farmácia do hospital, realizado por meio dos registos farmacêuticos de dispensação dos medicamentos. Relacionado a isso, é relevante esclarecer que participaram neste estudo pessoas com HIV/AIDS em tratamento e que, em Portugal, todas as pessoas portadoras de HIV e AIDS têm acesso gratuito à medicação antirretroviral. Deste modo, cada pessoa visita mensalmente à farmácia do hospital para levar a medicação para casa, sendo efetuado um registo informático do dia e da quantidade de antiretrovirais dispensados para um mês. Assim, foi possível avaliar o grau de adesão a retirada da medicação e obter uma porcentagem. Para o cálculo da porcentagem de adesão a retirada na farmácia, os participantes foram avaliados por um período de três meses coincidente com o preenchimento dos questionários.

Como pode ser identificado na Tabela 1 , o grau de adesão médio a retirada da medicação situa-se nos $91,1 \%$ $(D P=15,8 \%)$ e varia no intervalo de 25 a $100 \%$.

\section{Instrumentos}

Questionário de Dados Sociodemográficos e Clínicos. Incluiu informação acerca das características sociodemográficas idade, sexo, estado civil, escolaridade e ocupação; e clínicas, via de infecção, tempo de infecção, carga viral, número de linfócitos T CD4+ e terapêutica antirretroviral.

Questionário para a Avaliação da Adesão ao Tratamento Antirretroviral. O questionario CEAT-VIH como denominado no original (Remor, 2002a), é constituído por 20 itens para avaliar de maneira específica a adesão à terapêutica antirretroviral. É um instrumento de carácter multidimensional, de auto administração para indivíduos adultos. Constitui uma ferramenta rápida e simples de administrar, abarcando os principais fatores associados ao comportamento de adesão. Quanto maior a pontuação, maior será o grau de adesão ao tratamento 
Tabela 1

Caracterização dos Participantes em Função das Variáveis Clínicas. Porto, Portugal, 2007 (N=125)

\begin{tabular}{|c|c|c|c|c|c|c|}
\hline Variáveis clínicas & $M$ & $D P$ & Mín & Máx & $N$ & $(\%)$ \\
\hline \multicolumn{7}{|l|}{ Via de infecção } \\
\hline Sexual & & & & & 78 & 62,4 \\
\hline Sanguinea & & & & & 47 & 37,6 \\
\hline \multicolumn{7}{|l|}{ Tratamento Antirretroviral } \\
\hline 1INTR $*+1 \mathrm{INNTR} * *$ & & & & & 49 & 39,2 \\
\hline 2INTR+1INNTR & & & & & 20 & 16 \\
\hline $2 \mathrm{INTR}+1 \mathrm{IP} * * *$ & & & & & 22 & 17,6 \\
\hline 1INTR+1INNTR+1IP & & & & & 1 & 0,8 \\
\hline 1INTR+1IP & & & & & 25 & 20 \\
\hline 1INTR+2IP & & & & & 6 & 4,8 \\
\hline 2INTR+2IP & & & & & 2 & 1,6 \\
\hline Tempo de diagnóstico HIV-positivo (meses) & 69,5 & 41,6 & 4 & 170 & & \\
\hline Carga vírica (cópias/ml) & $2.029,8$ & $7.575,9$ & $<50$ & 57.698 & & \\
\hline$<50$ (indetectável) & & & & & & 70,4 \\
\hline $51-500$ & & & & & & 12,0 \\
\hline $501-10000$ & & & & & & 6,4 \\
\hline $10001-30000$ & & & & & & 7,2 \\
\hline$>30000$ & & & & & & 4,0 \\
\hline Linfócitos $T C D 4+$ & 359,7 & 243,1 & 14 & 1167 & & \\
\hline$<200$ & & & & & & 24,8 \\
\hline $200-350$ & & & & & & 30,4 \\
\hline $351-500$ & & & & & & 24,8 \\
\hline$>500$ & & & & & & 20 \\
\hline $\begin{array}{l}\text { \% Adesão ao levantamento da medicação } \\
\text { anti-retrovírica na Farmácia Hospitalar }\end{array}$ & $(0-100)$ & 91,1 & 15,8 & 25 & 100 & \\
\hline
\end{tabular}

Notas. * Inibidores Nucleósidos da Transcriptase Reversa; ** Inibidores Não Nucleósidos da Transcriptase Reversa; *** Inibidores da Protease.

antirretroviral (Remor, 2002a). O CEAT-VIH foi utilizado na sua forma adaptada para português de Portugal por Reis (2007). Estudos prévios confirmaram as propriedades psicométricas (fidedignidade, validade, sensibilidade e especificidade) do instrumento para diferentes países: Espanha (Remor, 2002a, 2008), Colômbia (Villa-González, 2005), Portugal (Reis, 2007; Reis, Lencastre, \& Guerra, 2007), Brasil (Remor, MilnerMoskovics, et al., 2007), Peru e México (Remor, 2008). A fidedignidade (alpha de Cronbach) para o presente estudo foi de 0,71 .

Questionário Breve de Qualidade de Vida da Organização Mundial de Saúde (WHOQOL-Bref). Constituise em uma versão reduzida (26 itens) do WHOQOL-100 (Fleck, 2006). Este instrumento conserva as 24 facetas do WHOQOL-100 e mantém a essência subjetiva e multidimensional do conceito de qualidade de vida (Vaz Serra et al., 2006). O WHOQOL-Bref está organizado em quatro domínios (entre parênteses apresentam-se os alphas de Cronbach neste estudo para cada domínio): Físico $(0,81)$, Psicológico $(0,85)$, Relações Sociais $(0,79)$ e Ambiente (0,81). Através da pontuação conjunta dos quatro domínios obtém-se uma pontuação geral que representa a qualidade de vida global do indivíduo. Uma maior pontuação em cada domínio significa maior qualidade de vida.

Inventário Breve de Sintomas (Brief Symptom Inventory, BSI). Os indicadores de psicopatologia foram avaliados por meio do BSI de Derogatis (1982), na sua versão adaptada e validada para Portugal por Canavarro (1999). O BSI é um inventário de auto-resposta com 53 itens, em que o indivíduo deve classificar o grau em que cada problema o afetou durante a última semana, numa escala tipo Likert que varia entre "Nunca" (0) a "Muitíssimas vezes" (4). As subescalas que compõem o BSI são (alphas de Cronbach entre parênteses para o presente estudo): Somatização $(0,81)$, Obsessões-Compulsões $(0,76)$, Sensibilidade Interpessoal $(0,65)$, Depressão $(0,84)$, Ansiedade $(0,80)$, Hostilidade $(0,74)$, Ansiedade Fóbica $(0,62)$, Ideação Paranóide $(0,64)$ e Psicoticismo $(0,56)$. Ademais, o inventário permite calcular três variáveis resumo: índice global de severidade (IGS), total de 
Reis, A. C., Lencastre, L., Guerra, M. P. \& Remor, E. (2010). Relação entre Sintomatologia Psicopatológica, Adesão ao Tratamento e Qualidade de Vida na Infecção HIV e AIDS.

sintomas positivos (TSP) e índice de sintomas positivos (ISP). Uma maior pontuação em cada dimensão indica maior presença de sintomas de disfunção psicológica.

\section{Procedimentos}

Os dados foram coletados durante os meses de Junho de 2006 e Fevereiro de 2007 na Unidade de consulta de Infectologia do Hospital de Joaquim Urbano na cidade do Porto (Portugal). Os participantes HIV-positivo, todos em tratamento antirretroviral há pelo menos três meses, preencheram o protocolo de pesquisa (questionários) ao final da consulta com o médico e sempre na presença da psicóloga responsável pelo estudo. Os dados relativos ao perfil clínico foram obtidos diretamente do historial médico dos pacientes. O estudo recebeu parecer favorável da Comissão de Ética e do Conselho de Administração do Hospital de Joaquim Urbano (Porto, Portugal). A participação foi voluntária e todos assinaram o Termo de Consentimento Livre e Esclarecido, pelo qual aceitavam as condições do estudo.

\section{Resultados}

Comparação entre Portadores de HIV e AIDS

e a População Geral em Relação à Sintomatologia

Psicopatológica e à Qualidade de Vida

Primeiramente, os dados foram avaliados mediante a comparação dos resultados dos participantes no estudo com HIV e AIDS $(n=125)$ aos da população geral $(n=$ 604) referentes ao estudo de adaptação portuguesa do WHOQOL-Bref realizado por Vaz Serra et al. (2006). Essa comparação indicou que os indivíduos HIV-positivo apresentam valores inferiores aos da população geral nos domínios da qualidade de vida [Físico $(t=-3,28, p=$ $0,001)$, Relações sociais $(t=-3,35, p=0,001)$, Ambiente $(t=-2,10, p=0,037)$, e no domínio Geral $(t=-5,25, p$ $=0,000)]$. O único domínio que não apresentou diferenças significativas entre HIV-positivo e população geral foi o Psicológico $(t=-0,72, p>0,05)$.

Após, para avaliar possíveis diferenças nos indicadores de disfunção psicológica avaliados pelo BSI, foi feita a comparação com os resultados obtidos por Canavarro (1999) referentes à população geral $(n=404)$ e os apresentados pelos participantes no presente estudo $(n=125)$. Os resultados indicaram que as pessoas com HIV e AIDS apresentam valores médios significativamente mais elevados em alguns indicadores de sintomatologia psicopatológica comparativamente à população geral sem problemas de saúde declarados. As dimensões nas quais os participantes HIV-positivo obtiveram maior pontuação foram: Somatização $(t=2,38, p=0,019)$ e Índice de Sintomas Positivos $(t=5,29, p=0,000)$. Verificou-se, ainda, que a pontuação média $(2,02)$ do Índice de Sintomas Positivos do BSI para a amostra HIV-positivo foi claramente superior ao ponto de corte de 1,7 definido pelo manual do instrumento. Este valor descreve a probabilidade de encontrar pessoas emocionalmente disfun- cionais na amostra estudada, tal como é avaliada pelo instrumento.

Além disso, encontrou-se significativamente maior pontuação média para a população geral frente aos participantes HIV-positivo nas dimensões: ObsessõesCompulsões $(t=-6,64, p=0,000)$, Sensibilidade interpessoal $(t=-2,76, p=0,007)$, e Total de Sintomas Positivos $(t=-4,33, p=0,000)$.

\section{Influência do Nível de CD4+ sobre os Indicadores} de Qualidade de Vida Avaliados pelo WHOQOL-Bref

Para avaliar a possível influência do nível de CD4+ (indicador inmunológico) sobre os domínios da qualidade de vida na amostra HIV-positivo, realizou-se uma análise (teste $t$ de Student) comparando os indivíduos HIV-positivo em função do nível de células CD4+. O ponto de corte foi 200 células T CD4+/ml. Os resultados mostraram que não há diferenças estatisticamente significativas entre os indivíduos HIV-positivo com menos de 200 células CD4+/ml (indicativo de AIDS) e os indivíduos com mais 200 células CD4+/ml.

\section{Relação entre Sintomatologia Psicopatológica,} Adesão ao Tratamento e Qualidade de vida na Infecção pelo HIV e AIDS

As correlações entre sintomatologia psicopatológica, adesão ao tratamento e qualidade de vida nos participantes deste estudo podem ser consultadas na Tabela 2 .

No que se refere à relação existente entre sintomatologia psicopatológica e adesão ao tratamento, observamse correlações negativas e significativas $(p<0,01)$ para as dimensões do BSI, à exceção do Índice geral de sintomas que não apresentou associação com a adesão ao tratamento. Estes resultados indicam que a maior frequência de sintomas psicopatológicos está relacionada a um menor nível de adesão ao tratamento.

Também verificou-se que a adesão ao tratamento avaliada pelo CEAT-VIH apresenta correlações positivas e significativas $(p<0,01)$ com todos os domínios da qualidade de vida, incluindo o domínio Geral. Estes resultados sugerem que os indivíduos com maior adesão ao tratamento também apresentaram maior qualidade de vida.

Por fim, os indicadores de sintomatologia psicopatológica apresentaram-se negativamente correlacionados aos domínios da qualidade de vida, indicando que a maior presença de sintomatologia psicopatológica se associa a menor qualidade de vida avaliada pelo WHOQOL-Bref.

\section{Modelo Preditor de Adesão Terapêutica na Infecção pelo HIV e AIDS}

Para identificar o modelo que melhor explica a adesão terapêutica na infecção HIV e AIDS foram incluídas no modelo de regressão as variáveis que estavam relacionadas com a variável de resultado grau de adesão ao tratamento medido pelo CEAT-VIH: total de sintomas 


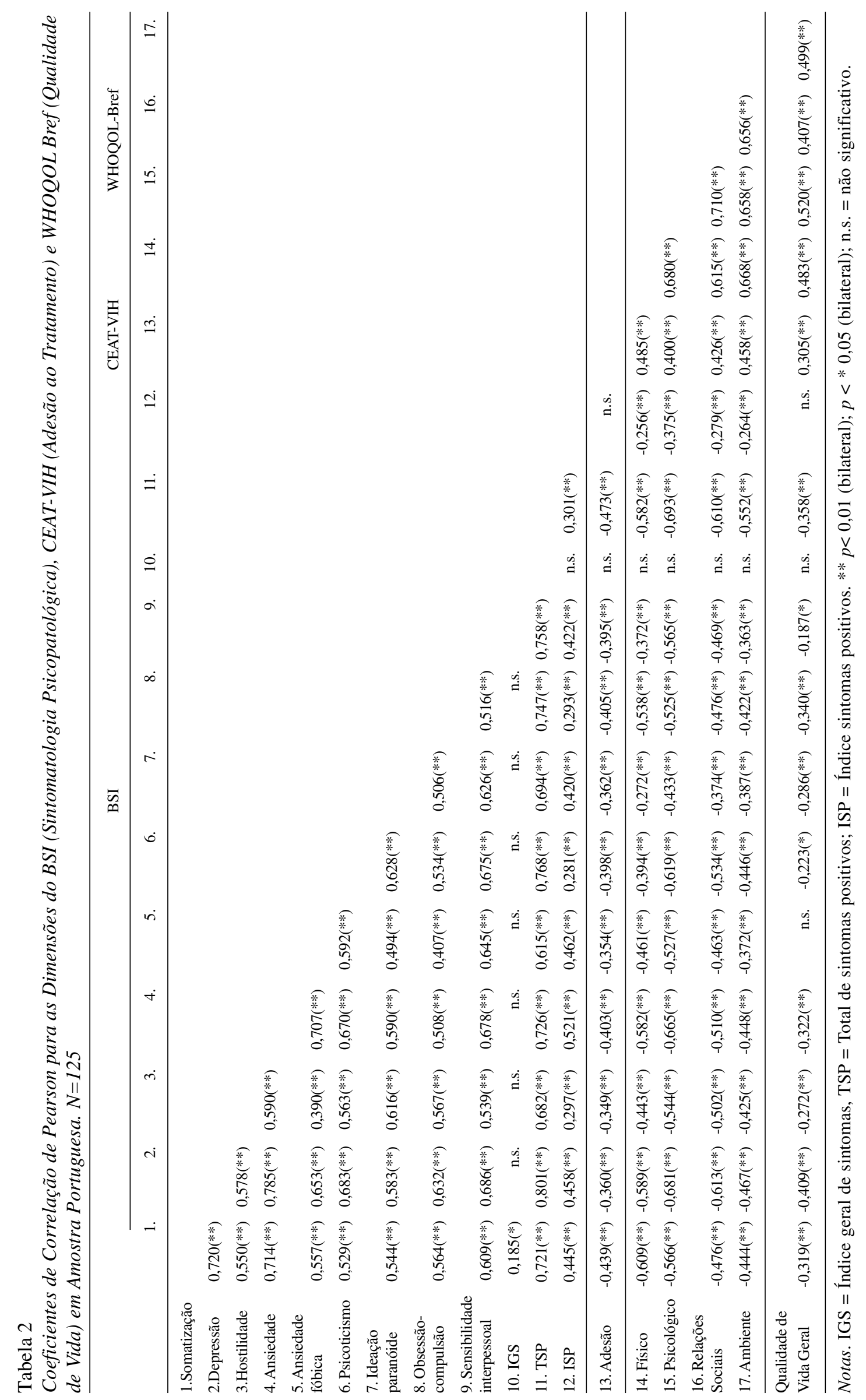


Reis, A. C., Lencastre, L., Guerra, M. P. \& Remor, E. (2010). Relação entre Sintomatologia Psicopatológica, Adesão ao Tratamento e Qualidade de Vida na Infecção HIV e AIDS.

positivos (índice do BSI), adesão à farmácia e total de efeitos secundários observados. A análise estatística foi realizada recorrendo ao procedimento de Regressão Linear Múltipla através do método Stepwise.

As três variáveis incluídas no modelo mostraram-se preditoras da adesão, explicando em conjunto $30,2 \%$ da variância total da adesão $\left(R^{2}=0,302 ; F[3,124]=17,476\right.$; $p=0,000)$.

Analisando individualmente cada variável verificouse que o TSP $(\beta=-0,435 ; p=0,000)$ é o fator que contribui em maior medida para a variância total da adesão (22\%). Neste caso concreto, a relação entre as duas variáveis é inversa, ou seja, quanto menor for a presença de sintomas positivos (psicopatológicos), maior será a adesão terapêutica. Por sua vez, a porcentagem de medicação antirretroviral retirada na Farmácia do Hospital $(\beta=0,246 ; p=0,002)$ representa o segundo fator que mais contribui para a adesão com $6 \%$ da variância, ou seja, os participantes que foram buscar a medicação com maior assiduidade à farmácia apresentaram maior adesão ao tratamento. E finalmente, o somatório da experiência de efeitos colaterais $(\beta=0,162 ; p=0,049)$ é o terceiro e último factor que permite predizer a adesão, contribuindo com $2 \%$ da variância. Como se pode observar, a relação entre as variáveis é positiva, o que sugere que uma maior experiência de efeitos colaterais associados à medicação antirretroviral significa maior adesão ao tratamento.

\section{Efeito Mediador da Sintomatologia Psicopatológica} (TSP) entre a Adesão ao Tratamento e a Qualidade de Vida na Infecção pelo HIV e AIDS

Foi efetuada uma análise de correlação de Pearson que permitiu identificar a relação existente entre: (a) adesão ao tratamento e qualidade de vida medida pelo domínio Geral do WHOQOL-Bref $(r=-0,30 ; p=0,001), 2)$, total de sintomas positivos (TSP) e qualidade de vida geral $(r$ $=-0,35 ; p=0,000)$, e 3 ) adesão e TSP $(r=-0,47 ; p=$ $0,000)$. Não foram observadas relações estatisticamente significativas entre as outras variáveis principais e a qualidade de vida. A análise de regressão linear múltipla (através do método Stepwise) para a qualidade de vida identificou que apenas o TSP $(\beta=-0,358 ; p=0,000)$ foi preditor da qualidade de vida $\left(R^{2}=0,128 ; F=18,095\right.$; $p=0,000)$, sendo excluída do modelo a variável adesão ao tratamento. De acordo com esta análise, é possível afirmar que a presença de sintomas positivos (psicopatológicos) pressupõe uma deterioração na qualidade de vida das pessoas portadoras de HIV e AIDS, independentemente do grau de adesão ao tratamento antirretroviral.

Considerando a importância do tratamento antirretroviral e da adesão ao tratamento para a manutenção da qualidade de vida em pessoas vivendo com o HIV e AIDS referida na literatura especializada (Remor et al., 2008), buscou-se testar a hipótese de que a relação entre adesão ao tratamento e qualidade de vida poderia estar mediada pela presença de sintomas positivos (TSP). Para isso, foi realizada uma análise de regressão hierárquica para o teste de mediação seguindo o modelo de Baron e Kenny (1986) que refere que o mediador se define como uma variável que media a relação entre uma variável preditora e uma variável de resultado, ou seja, um mediador é o mecanismo através do qual o preditor influencia a variável de resultado. O modelo hipotético de mediação é apresentado na Figura 1.

De acordo com este método, há quatro passos (realizados com três equações de regressão) para estabelecer que uma variável (p. ex. total de sintomas positivos) media a relação entre a variável preditora (p. ex. adesão ao tratamento) e a variável de resultado (p. ex. qualidade de vida geral). O primeiro passo é mostrar que há uma relação significativa entre o preditor e a variável de resultado (ver Path c na Figura 1, parte 1A). O segundo passo é mostrar que o preditor está relacionado com o mediador (ver Path a na Figura 1, parte 1B). O terceiro passo é mostrar que o mediador (p. ex. total de sintomas positi-

$1 \mathrm{~A}$.

\begin{tabular}{|l|l|}
\hline $\begin{array}{l}\text { Preditor } \\
\text { Adesão ao tratamento }\end{array}$ & Path \\
Vualiável de Resultado de Vida Geral \\
Qualidade de
\end{tabular}

$1 B$.

Path c'

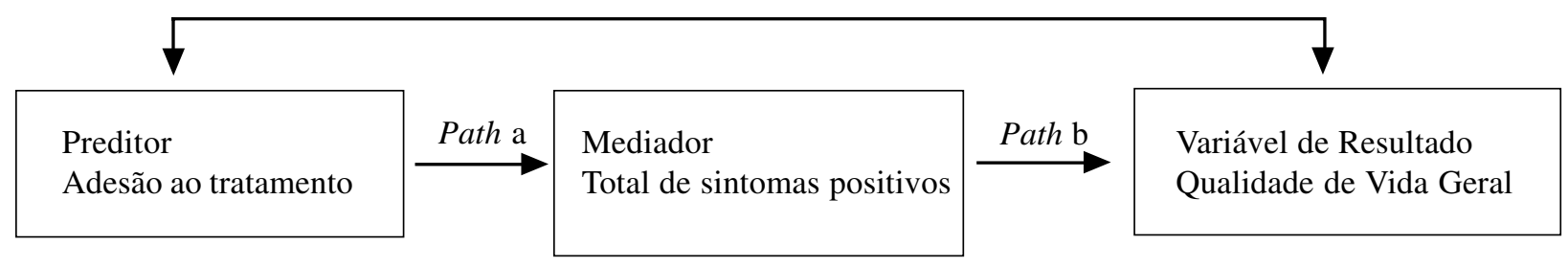

Figura 1. Diagrama dos "Paths" para o modelo hipotético de mediação de acordo com Baron e Kenny (1986) 
vos) está relacionado com a variável de resultado (p. ex. qualidade de vida geral). Este é o Path b na Figura 1, parte 1B, que se estima ao controlar-se o efeito do TSP na variável de resultado. O passo final é mostrar que a força da relação entre o preditor e a variável de resultado é reduzida (ou anulada) significativamente quando o mediador é incluído no modelo de regressão (comparar o Path c na Figura 1A com o Path c' na Figura 1, parte
1B). Se o "total de sintomas positivos" é um mediador, a relação entre adesão ao tratamento e qualidade de vida deixará de ser significativa quando o TSP é incluído no modelo de regressão.

Os resultados relativos a esta análise são apresentados com detalhe na Tabela 3. Estes resultados sugerem um efeito de mediação do total de sintomas positivos entre adesão ao tratamento e qualidade de vida geral.

Tabela 3

Teste de Mediação: Efeito de Mediação do Total de Sintomas Positivos entre a Adesão ao Tratamento e a Qualidade de Vida

\begin{tabular}{lrrrr}
\hline & B & SE B & 95\% IC & â \\
\hline $\begin{array}{l}\text { Teste passo 1 (Path c) } \\
\text { Variável de resultado: Qualidade de Vida Geral }\end{array}$ & & & & \\
$\begin{array}{l}\text { Variável preditora: Adesão ao tratamento } \\
\text { Teste passo 2 (Path a) }\end{array}$ & 0,079 & 0,022 & 0,$035 ; 0,123$ & $0,305^{* *}$ \\
$\begin{array}{l}\text { Variável de resultado: Total de sintomas positivos (BSI) } \\
\text { Variável preditora: Adesão ao tratamento }\end{array}$ & $-0,986$ & 0,166 & $-1,314 ;-0,657$ & $-0,473^{* *}$ \\
$\begin{array}{l}\text { Teste passo 3 (Paths b \& c') } \\
\text { Variável de resultado: Qualidade de Vida Geral }\end{array}$ & & & & \\
$\begin{array}{l}\text { Mediador: Total de sintomas positivos (BSI) } \\
\text { Preditor: Adesão ao tratamento }\end{array}$ & & & & \\
Bloco 1 (Path b) & & & & \\
Total de sintomas positivos (mediador) & & & \\
Bloco 2 (Path c') & $-0,044$ & 0,010 & $-0,065 ;-0,024$ & $-0,358^{* *}$ \\
Total de sintomas positivos (mediador) & & & & \\
Adesão ao tratamento (preditor) & $-0,034$ & 0,012 & $-0,057 ;-0,011$ & $-0,276^{* *}$ \\
\hline
\end{tabular}

Notas. IC $=$ Intervalo de confiança; $* * p<0,001 ;$ n.s. = não significativo $; \hat{a}=$ Coeficiente de regressão estandarizado.

\section{Discussão}

O perfil das pessoas com HIV e AIDS incluídos neste estudo foi predominantemente constituído por participantes do sexo masculino, com idade média não superior aos 40 anos, com baixo nível de escolaridade, empregados, e infectados por via sexual. Estudos realizados no Brasil (Melchior, Nemes, Alencar, \& Buchella, 2007; Santos, França, \& Lopes, 2007) descreveram um perfil semelhante ao da nossa amostra em Portugal.

Apesar de as pessoas com HIV avaliadas neste estudo cumprirem em boa parte os critérios para o diagnóstico de AIDS, na data do preenchimento dos questionários, a maioria apresentava valores de carga viral "indetectáveis", indicando que a infecção pelo HIV estava controlada. Este dado sugere a possibilidade de que, talvez, o perfil sociodemográfico não seja determinante para o comportamento de autocuidado com a saúde, e que provavelmente a variabilidade nas diferenças individuais observadas possam ser explicadas por outras variáveis mais relacionadas com o contexto, qualidade do tratamento prescrito ou recursos psicológicos desenvolvidos pelo indivíduo. Neste sentido, o trabalho prévio de
Remor et al. (2008) apontava que as características sociodemográficas e o nível socioeconômico não estavam relacionados com o cumprimento do tratamento antirretroviral em uma amostra brasileira.

As variáveis que foram alvo de atenção neste estudo revelaram-se importantes para a compreensão de outros possíveis fatores associados à adesão terapêutica à medicação antirretroviral, por exemplo, observou-se a relação dos indicadores de disfunção emocional avaliados pelo BSI aos baixos níveis de adesão ao tratamento. Estudos prévios observaram resultados similares utilizando outros indicadores de disfunção emocional como, por exemplo, medidas de ansiedade, estresse e depressão (Campos et al., 2008; Reis, 2007; Remor, 2002a; Remor et al., 2008).

De maneira similar, os indicadores de sintomatologia psicológica avaliados pelo BSI estão relacionados a uma menor percepção de qualidade de vida, sugerindo que a disfunção psicológica pode constituir um fator de risco para a qualidade de vida (associações negativas), enquanto a adesão ao tratamento se mostrou um fator de proteção para a qualidade de vida (associações positivas). Estudos realizados no Brasil identificaram relações 
Reis, A. C., Lencastre, L., Guerra, M. P. \& Remor, E. (2010). Relação entre Sintomatologia Psicopatológica, Adesão ao Tratamento e Qualidade de Vida na Infecção HIV e AIDS.

similares (Giovelli, 2009; Seidl, Zannon, \& Tróccoli, 2005).

Por outro lado, os resultados deste trabalho alertam que a soropositividade ao HIV não supõe um impacto negativo na saúde mental do indivíduo (passada a etapa de adaptação ao diagnóstico prevista entre seis e oito semanas; Remor, 2003b) se este apresentar recursos psicológicos e de apoio social para lidar com a situação. De fato, a amostra de pessoas com HIV/AIDS selecionada para este estudo (entre quatro e 170 meses de tempo de infecção) apresentou menores índices de indicadores psicopatológicos avaliados pelo BSI em comparação com a população geral (dados coletados por Canavarro, 1999), exceto para as dimensões Somatização e Índice de sintomas positivos. Assumindo que a infecção pelo HIV é uma doença de curso mais ou menos prolongado que culmina com uma destruição praticamente completa dos Linfócitos CD4+, e com períodos de emergência de sintomas associados a doenças oportunistas, porém tratável eficazmente com a medicação antirretroviral disponível (Blanco, Mallolas, \& Gatell, 2007), supõe-se que certo grau de preocupação ou vigilância com qualquer sintoma inespecífico (indicadores avaliados pela escala de Somatização do BSI) pode estar presente, bem como a experiência de Distresse emocional associado a esta preocupação (indicador avaliado pela escala de Índice de Sintomas Positivos do BSI, Positive Symptom Distress Index no original, Derogatis, 1982).

Outro resultado de interesse são as diferenças observadas na qualidade de vida comparando pessoas com HIV e AIDS e população geral. A infecção pelo HIV supõe uma deterioração nas dimensões física, social e ambiental da qualidade de vida das pessoas HIV-positivo, porém não na dimensão psicológica. Estes resultados apóiam a idéia referida no parágrafo anterior e foram, de maneira similar, descritos por Maia (2006) e Reis (2007).

Ao comparar subgrupos dentro da amostra HIV-positivo, observa-se que a deterioração na saúde representada pelo declínio imunológico (ponto de corte 200 células T CD4+/ml) parece não contribuir em diferenças na qualidade de vida percebida. Em um estudo brasileiro desenvolvido por Santos et al. (2007) que avaliou a qualidade de vida de portadores de HIV através do WHOQOL-Bref, também não foram encontradas diferenças estatisticamente significativas nos domínios do instrumento em função das células CD4+. De acordo com a literatura especializada prévia (Remor, 2003b, 2005; Remor, Young, von Mackensen, \& Lopatina, 2004), os instrumentos genéricos de qualidade de vida, por exemplo, o WHOQOL-Bref, são úteis na comparação entre diferentes doenças ou com a população geral sem problema de saúde declarado, mas não são sensíveis na detecção de mudanças ou diferenças na condição de saúde de uma população clínica específica. Para isso, são recomendados os instrumentos de avaliação da qualidade de vida específicos, por exemplo, o WHOQOL-HIVBref, que inclui perguntas relevantes para o problema de saúde em questão. Futuros trabalhos deverão considerar este aspecto no momento de escolher as medidas de avaliação.

O modelo preditivo que melhor explica o comportamento de adesão terapêutica contempla o índice Total de Sintomas Positivos (TSP) avaliado pelo BSI, o grau de adesão dos participantes aos levantamentos da medicação antirretroviral na farmácia do hospital e o somatório dos efeitos colaterais experienciados pelos participantes. Analisam-se cada uma destas variáveis em particular.

O TSP representa o número de queixas somáticas apresentadas, ao contrário do ISP que constitui uma medida da percepção da intensidade dos sintomas (distresse). Estudos anteriores (Gordillo, del Amo, Soriano, \& González-Lahoz, 1999) relacionaram a instabilidade emocional e social e indicadores psicopatológicos com baixa adesão ao tratamento.

O comportamento voluntário de ir à farmácia do hospital, seguindo assim as prescrições do médico, reflete a intenção por parte do indivíduo para assumir o comportamento de adesão. $\mathrm{O}$ fato dos indivíduos irem à farmácia do hospital expressa a primeira etapa da motivação para aderir ao tratamento prescrito, provavelmente consequência da boa relação técnico de saúde/paciente que facilita a aproximação dos indivíduos a este estabelecimento para levar a medicação para casa, constituindo-se em um comportamento de saúde. Estudos anteriores relacionaram indicadores similares como a assistência às consultas médicas marcadas ou ao centro de saúde, com o grau de adesão ao tratamento (Holzemer et al., 1999; McClure, Catz, \& Brantley, 1999).

O somatório dos efeitos colaterais da medicação antirretroviral informados pelos participantes deste estudo constitui também um preditor de adesão ao tratamento. Este fator foi citado anteriormente na literatura como preditor de baixa adesão ou interrupção do tratamento devido ao fato dos efeitos colaterais associados à medicação antirretroviral serem intensos e complexos, levando alguns pacientes a abandonar o tratamento (Berg et al., 2004; Remien et al., 2003). No presente estudo, encontrou-se uma relação positiva entre adesão ao tratamento e a presença de efeitos colaterais coadjuvantes ao tratamento. Esta relação aponta uma perspectiva diferente, isto é, pode-se entender que só aqueles que cumprem o tratamento assiduamente experienciam efeitos colaterais no decurso do tratamento. É possível que estes efeitos colaterais dos tratamentos mais modernos e atuais não sejam tão intensos, permitindo que o paciente siga o tratamento apesar dos possíveis efeitos adversos do medicamento.

Por último, parece importante referir o efeito mediador da sintomatologia psicopatológica (TSP) entre a adesão ao tratamento e a qualidade de vida. A presença de disfunção emocional parece minimizar o efeito da adesão ao tratamento na qualidade de vida. A literatura prévia relaciona o uso adequado da medicação antirretroviral (e adesão) como um fator de proteção para uma 
boa saúde e qualidade de vida na população com infecção pelo HIV e AIDS. Porém, observou-se no presente estudo que na presença de sintomas psicopatológicos a qualidade de vida diminui independentemente da adesão ao tratamento do indivíduo. Estudos prévios (Remor, Penedo, et al., 2007), numa perspectiva similar, observaram que o estresse, fator associado à precipitação de psicopatologia (Dohrenwend, 1998), atuava como fator de risco para a deterioração imunológica de pessoas com HIV e AIDS ao fim de seis meses de acompanhamento, independentemente do tempo e tipo de tratamento antirretroviral recebido, quantidade de vírus (carga viral) na linha base, quantidade de células T CD4+ na linha base, e grau de adesão ao tratamento. Portanto, o estresse acelerava a deterioração imunológica, independentemente das variáveis relacionadas com a infecção pelo HIV e o tratamento. Considerando os resultados do estudo atual, parece importante detectar precocemente possíveis indicadores de sintomas de disfunção emocional com o objetivo de paliá-los ou tratá-los psicologicamente para que estes não cheguem a influenciar negativamente a adesão ao tratamento e a qualidade de vida das pessoas com HIV e AIDS.

\section{Referências}

Baron, R. M., \& Kenny, D. A. (1986). The moderator-mediator variable distinction in social psychological research: Conceptual, strategic and statistical considerations. Journal of Personality and Social Psychology, 51(6), 1173-1182.

Berg, K., Demas, A., Howard, A., Schoenbaum, E., Gourevitch, M., \& Arnsten, J. (2004). Gender differences in factors associated with adherence to Antiretroviral Therapy. Journal of General Internal Medicine, 19, 1111-1117.

Blanco, J. L., Mallolas, J., \& Gatell, J. M. (2007). Historia natural, clasificación y pronóstico de la infección por VIH1. In J. M. Gatell, B. Clotet, D. Podzamczer, J. M. Miró, \& J. Mallolas (Eds.), Guía práctica del SIDA: clínica, diagnóstico y tratamiento (9. ed., pp. 49-61). Barcelona, Espanã: Elsevier Masson.

Campos, L. N., Guimarães, M. D., \& Remien, R. H. (2008). Anxiety and depression symptoms as risk factors for nonadherence to antiretroviral in Brazil. Aids and Behavior, 18(6), 529-536.

Canavarro, M. C. (1999). Inventário de Sintomas Psicopatológicos - B. S. I. In M. R. Simões, M. Gonçalves, \& L. S. Almeida (Eds.), Testes e provas psicológicas em Portugal (Vol. 2, pp. 95-109). Braga, Portugal: APPORT.

Canavarro, M. C., Pereira, M., Simões, M., Pintassilgo, A., \& Ferreira, A. (2008). Estudos psicométricos da versão portuguesa (de Portugal) do Instrumento de Avaliação da Qualidade de Vida na Infecção VIH da Organização Mundial de Saúde (WHOQOL-HIV). Psicologia, Saúde \& Doenças, 9(1), 15-28.

Carrobles, J. A., Remor, E., \& Rodríguez-Alzamora, L. (2003) Afrontamiento, apoyo social percibido y distrés emocional en pacientes con Infección por VIH. Psicothema, 15(3), 420-426.

Derogatis, L. R. (1982). BSI: Brief Symptom Inventory. Minneapolis, MN: National Computers Systems.
Dohrenwend, B. P. (1998). Adversity, stress and psychopathology. New York: Oxford University Press.

Fleck, M. A. (2006). O Projecto WHOQOL: Desenvolvimentos e aplicações. Psiquiatria Clínica, 27(1), 5-13.

Giovelli, G. R. M. (2009). Relação entre depressão, suporte social, qualidade de vida e adesão ao tratamento em pessoas que vivem com HIV/AIDS. Dissertação de Mestrado não-publicada, Faculdade de Psicologia, Pontifícia Universidade Católica do Rio Grande do Sul, Porto Alegre, RS.

Gordillo, V., del Amo, J., Soriano, V., \& González-Lahoz, J. (1999). Sociodemographic and psychological variables influencing adherence to antiretroviral therapy. AIDS, 13, 1763-1769.

Grilo, A. M. (2001). Temáticas centrais implicadas na vivência da infecção por VIH/SIDA. Psicologia, Saúde \& Doenças, 2(2), 101-118.

Guerra, M. P. (1998). SIDA. Implicações psicológicas. Lisboa, Portugal: Fim de Século.

Holzemer, W. L., Corless, I. B., Nokes, K. M., Turner, J. G., Brown, M. A., Powell-Cope, G. M., et al. (1999). Predictors of self-reported adherence in persons iving with HIV desease. AIDS Patient Care STDS, 13(3), 185-197.

Maia, C. M. (2006). Avaliação da qualidade de vida de portadores de HIV/Aids. Dissertação de Mestrado não-publicada, Universidade Católica de Goiás, Goiânia, GO.

McClure, J. B., Catz, S. L., \& Brantley, P. J. (1999). Early appointment adherence among persons living with HIV. AIDS and Behavior, 3(2), 85-176.

Melchior, R., Nemes, M., Alencar, T., \& Buchella, C. (2007). Desafios da adesão ao tratamento de pessoas vivendo com HIV/AIDS no Brasil. Revista de Saúde Pública, 41(2), 87-93.

Reis, A. (2007). Adesão terapêutica na infecção pelo vírus da imunodeficiência humana. Dissertação de Mestrado nãopublicada, Faculdade de Psicologia e de Ciências da Educação, Universidade do Porto, Portugal.

Reis, A., Lencastre, L., \& Guerra, M. (2007, July). Portuguese adaptation of the "Questionnaire to Assess Adherence to Antirretroviral Treatment - HIV”. Symposium hispano-luso de Medicina Conductual y Psicología de la Salud, Madrid, España.

Remien, R., Hirky, E., Johnson, M., Weinhardt, L., Whittier, D., \& Minh Le, G. (2003). Adherence to medication treatment: A qualitative study of facilitators and barriers among diverse sample of HIV-positivo men and women in Four U. S. Cities. AIDS and Behavior, 17(1), 61-72.

Remor, E. (1999). Abordagem psicológica da AIDS através do enfoque cognitivo-comportamental. Psicologia: Reflexão $e$ Crítica, 12(1), 89-106.

Remor, E. (2002a). Valoración de la adhesión al tratamiento antirretroviral en pacientes VIH+. Psicothema, 14(2), 262267.

Remor, E. (2002b). Apoyo social y calidad de vida en la infección por VIH. Atención Primaria, 30(3), 143-148.

Remor, E. (2003a). Fiabilidad y validez de la versión española del cuestionario MOS-SF-30 para evaluar la calidad de vida de personas infectadas con el VIH. Atención Primaria, 31, 15-22.

Remor, E. (2003b). Intervención del psicólogo en una unidad de tratamiento de pacientes con infección por VIH/SIDA. In E. Remor, P. Arranz, \& S. Ulla (Eds.), El psicólogo en el ámbito hospitalario (pp. 309-348). Bilbao, España: DDB. 
Reis, A. C., Lencastre, L., Guerra, M. P. \& Remor, E. (2010). Relação entre Sintomatologia Psicopatológica, Adesão ao Tratamento e Qualidade de Vida na Infecção HIV e AIDS.

Remor, E. (2005). Desarrollo de una medida específica para la evaluación de la calidad de vida en pacientes adultos viviendo con hemofilia en América-Latina: el HemoLatinQoL. Revista Interamericana de Psicología, 39(2), 211-220.

Remor, E. (2008, September). International Psychometric Study of the Adherence to Antiretroviral Treatment Questionnaire. Paper presented at the meeting of the European Health Psychology Conference, Bath, UK.

Remor, E., Ballester-Arnal, R., Espada-Sánchez, J. P., \& Gordillo Álvarez-Valdés, M. V. (2008). La investigación e intervención psicológicas en el ámbito de la infección por VIH en España: una revisión. In J. A. Piña-López \& J. J. Sánchez-Sosa (Eds.), Aportaciones desde la psicología a la infección por VIH: investigación e intervención (pp. 185215). México, DF: Plaza y Valdés.

Remor, E., Milner-Moskovics, J., \& Preussler, G. (2007). Estudo psicométrico para a adaptação brasileira do "Cuestionario para la Evaluación de la Adhesión al Tratamiento Antirretroviral (CEAT-VIH)". Revista de Saúde Pública, 41(5), 685-694.

Remor, E., Penedo, F. J., Shen B. J., \& Schneiderman, N. (2007). Perceived stress is associated with CD4+ cell decline in men and women living with HIV/AIDS in Spain. AIDS Care, 19(2), 215-219.

Remor, E., Young, N. I., von Mackensen, S., \& Lopatina, E. G. (2004). Disease-specific quality of life measurement tools for haemophilia patients. Haemophilia, 10(Suppl. 4), 30-34.

Santos, E. C. M., França, I., Jr., \& Lopes, F. (2007). Qualidade de vida de pessoas vivendo com HIV/AIDS em São Paulo. Revista de Saúde Pública, 41(2), 64-71.

Serovich, J. M., Kimberly, J. A., Mosack, K. E., \& Lewis, T. L. (2001). The role of family and friend social support in reducing emotional distress among HIV-positive women. AIDS Care, 13(3), 335-341.

Seidl, E. M., Zannon, C. M., \& Tróccoli, B. T. (2005). Pessoas vivendo com HIV e AIDS: Enfrentamento, suporte social e qualidade de vida. Psicologia: Reflexão \& Crítica, 18(2), 188-195.

Siegel, K., \& Lekas, H. M. (2002). AIDS as a chronic illness: Psychosocial implications. AIDS, 16, 69-76.

Vaz Serra, A., Canavarro, M. C., Simões, M., Pereira, M., Gameiro, S., Quartilho, M. J., et al. (2006). Estudos psicométricos do Instrumento de Avaliação da Qualidade de Vida da Organização Mundial de Saúde (WHOQOL-Bref) para Português de Portugal. Psiquiatria Clínica, 27(1), 41-49.

Villa-González, I. C. (2005). Calidad de vida, adhesión terapéutica y variables psicosociales moduladoras en personas que conviven con el VIH. Unpublished master's thesis, Facultad de Psicología, Universidad de San Buenaventura, Medellín, Colombia. 